\section{CRITICAL MEDICAL AND SURGICAL NURSING JOURNAL \\ Vol. 10, No. 2, October 2021}

Journal Homepage: https://e-journal.unair.ac.id/CMSNJ

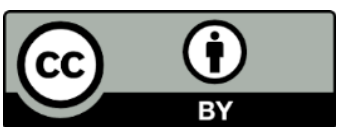

This is an Open Access article distributed under the terms of the Creative Commons Attribution 4.0 International License

\title{
Differences in the Characteristics of Ischemic and Hemorrhage Stroke Patients with Mechanical Phlebitis
}

\author{
Yeni Siswanti ${ }^{1}$, Ika Yuni Widyawati ${ }^{2} \mathbb{D}$, Candra Panji Asmoro $^{2}$ \\ ${ }^{1}$ Bachelor Student, Faculty of Nursing, Universitas Airlangga, Surabaya, Indonesia \\ ${ }^{2}$ Faculty of Nursing, Universitas Airlangga, Surabaya, Indonesia
}

\author{
ARTICLE HISTORY \\ Received: May 1, 2021 \\ Accepted: December 10, 2021 \\ Published: December 10, 2021
}

\section{KEYWORDS}

Characteristics,; Phlebitis; Hypertension; Diabetes

mellitus; Stroke; Pneumonia

\section{CORRESPONDING AUTHOR}

Ika Yuni Widyawati

ika-yw@fkp.unair.ac.id

Faculty of Nursing, Universitas

Airlangga, Surabaya, Indonesia

Cite this as:

\begin{abstract}
Introduction: One of the complications of stroke patients receiving intravenous therapy is mechanical phlebitis. This study aimed to analyze the differences characteristic of stroke patients who experienced mechanical phlebitis.

Methods: This study used a comparative descriptive method with a documentation study design. The subjects of this study were stroke patients who experienced mechanical phlebitis from January 2018 until September 2020. The sampling method used total sampling with 92 samples. The data analysis using the Chi-squared test.

Results: Gender ( $p=0.020)$ significantly differed with the incidence of mechanical phlebitis in stroke patients. Characteristics of age $(p=0.511)$, history of hypertension $(\mathrm{p}=0.058)$, diabetes mellitus $(\mathrm{p}=0.503)$, pneumonia $(\mathrm{p}=0.164)$, and stroke $(\mathrm{p}=0.160)$ did not have a significant difference with the incidence of mechanical phlebitis in stroke patients.

Conclusion: There are differences in gender characteristics in stroke patients who experience mechanical phlebitis. It is necessary to develop a phlebitis surveillance document that is equipped with insertion area, cannula material, the reason for infusion release, venous condition, installation length, insertion frequency, and observation of each shift accompanied by documentation control.
\end{abstract}

Siswanti, Y., Widyawati, I. Y., \& Asmoro, C. P. (2021). Differences in the Characteristics of Ischemic and Haemorrhage Stroke Patients with Mechanical Phlebitis.Crit. Méd. Surgical. nurse. J, 10(2), 57-61.

\section{INTRODUCTION}

Stroke is an acute neurological deficit disease which is a major cause of morbidity, mortality, and disability worldwide (Torres-Aguila, 2019). Appropriate management of acute stroke can minimize disability. Administration of crystalloid fluids (except hypotonic) and intravenous therapy via peripheral infusion is one of the treatments for hemodynamic stabilization of the patient. One of the complications of peripheral infusion is the occurrence of phlebitis which can be caused by mechanical, chemical, bacteremia or post-infusion factors. As much as $31 \%$ of patients who had peripheral infusions had phlebitis (Simin, 2019).

Mechanical phlebitis is an inflammation of the vein, which is characterized by pain, redness, the local area of insertion feels warm, swelling, appear induration, purulence, and hard palpable veins. Visual Infusion Phlebitis (VIP) Score is used to assess of the incidence of phlebitis. The VIP score zero indicates no symptoms phlebitis, while a score of one to five shows signs and symptoms phlebitis according to the parameters. Score two reported as incident phlebitis. (Gorski, 2016)

Incident of phlebitis in developing countries is still above the standard, according to the Center of Disease Control and Prevention (CDC) which recommend is $<5 \%$. According to Siregar (2019) phlebitis incident in India reach $27.91 \%$, Iran (14.20\%), Malaysia (12.70\%), Philippines (10.10\%) and Indonesia (9.80\%). The results of the preliminary study by researchers through document tracing on the surveillance report of the Infection Prevention and Control Committee at the Kanjuruhan Hospital, Malang Regency in January 2021, obtained the 
average incident rate data mechanical phlebitis of is $5.93 \%$.

The impact of untreated phlebitis is the occurrence of primary bloodstream infections (bacteremia) which can increase morbidity, mortality, prolong the healing process (Ying, 2020). Research conducted by Blanco-Mavillard (2019) states that complications of phlebitis can affect patient safety and increase the workload of health workers, especially nurses. Patient with phlebitis can experience delays in administering drugs via intravenous, longer treatment period, and increased hospital costs. Phlebitis become an unpleasant experience and lead to patient dissatisfaction. (Maier, 2019).

According to Eternal (2013), mechanical phlebitis caused by the size and diameter of the cannula, the material of the cannula, the duration of infusion and the skill of the staff at the time of inserting the infusion. Factors that influence venous wall trauma are the placement of a large cannula in a small diameter vein, insertion in the flexion area, stiffness of the cannula and inadequate stabilization technique. Host characteristics that increase the risk of occurrence mechanical phlebitis factors are fragile veins, hypercoagulable states, increased hemoglobin, ageing, gender, history of diabetes mellitus, infectious diseases, cancer, immunosuppression, circulatory disorders (Phillips \& Gorski, 2014).

The results of the preliminary study by researchers through the surveillance report of the Infection Prevention and Control Committee and medical records at the Kanjuruhan Hospital, Malang Regency in January 2021, obtained data that most of the patients undergoing inpatient care at the Kanjuruhan Hospital, Malang Regency in 2020 were diagnosed with stroke. Ischemic stroke is more common in patients aged over 45 years and female, while hemorrhagic stroke is more common in patients aged over 60 years. Stroke patients who received intravenous therapy, $2.98 \%$ experienced mechanical phlebitis. The incident of mechanical phlebitis in ischemic stroke six times more than hemorrhagic stroke.

Based on the epidemiological triangle theory proposed by Gordon and La Richt, it states that the incidence of intravenous wound infection or phlebitis associated with peripheral infusion is caused by an imbalance of interactions between host (host), agent (causative factor) and environment (environment). (Unahalekhaka, 2016). To improve the quality and safety of patients in hospitals, nurses have the responsibility to prevent this from phlebitis. Increasing the knowledge and competence of nurses regarding factors that increase the risk of phlebitis and intravenous therapy is one way to reduce the incidence of mechanical phlebitis in stroke patients.

\section{METHOD}

This study is a comparative descriptive study with a cohort study approach to determine the differences in the characteristics of ischemic stroke and hemorrhagic stroke patients with mechanical phlebitis. This research was conducted at Kanjuruhan Hospital, Malang Regency in January 2021. Permission for ethical clearance obtained from the Health Research Ethics Committee of Kanjuruhan Hospital (Number: 072.1/EA.KEPK$011 / 35.07 .208 / 2021)$. Amount the sample in this study was 92 people. The inclusion criteria in this study were stroke patients undergoing inpatient treatment at Kanjuruhan Hospital, Malang Regency and confirmed phlebitis mechanics in the Surveillance Report of the Infection Prevention and Control Committee in January 2018 to September 2020. The exclusion criteria in this study were 1) patients treated in intensive care, neonatal intensive care and children 2) patients undergoing blood cultures.

Data collection is done by recording events phlebitismechanics of stroke patients based on the surveillance report of the Infection Prevention and Control Committee from January 2018 to September 2020. The next step is to look for the medical record status of the respondent at the Medical Record and Evaporative Installation based on the registered register number. Data on age, gender, medical diagnoses established by the doctor in charge of the patient, history of circulatory disorders, history of immunosuppression and history of accompanying infectious diseases are recorded based on medical record documents. The data is then recapitulated using the observation sheet in the software.

The results of the study were analyzed using univariate and bivariate statistical tests. The univariate analysis used in this study is a descriptive analysis of the characteristics of the respondents, namely the type of stroke, age, gender, history of hypertension, diabetes mellitus, pneumonia, and stroke. Bivariate analysis was tested with Chi-square test which aims to determine the differences in characteristics between ischemic stroke patients and hemorrhagic patients who have mechanical phlebitis.

\section{RESULT}

In table 1 , it was obtained information that the ischemic stroke respondents experienced more mechanical ventilation (60.9\%) compared to hemorrhagic stroke. The distribution of respondents who experienced mechanical phlebitis was mostly at the age of $>66$ years (41.3\%). Most of the respondents had a history of hypertension (58.7\%). The incidence of mechanical phlebitis was more experienced by respondents who did not have a history of diabetes mellitus (90.2\%), did not suffer from infectious diseases (pneumonia) (95.6\%) and did not have a history of stroke (73.9\%).

From table 2, the results of bivariate analysis show that there is one independent variable, namely gender which has a significant value $(p=0.020)$ on the incidence of mechanical phlebitis. Variable age $(p=0,511)$, history of hypertension $(p=0,058)$, 
diabetes mellitus $(0,503)$, pneumonia $(p=0,164)$, and stroke $(p=0,160)$ did not show a significant value to the incidence of mechanical phlebitis.

\section{DISCUSSION}

From the results of the study, it was found that there was no difference in the characteristics of age on the incidence ofphlebitis mechanics between ischemic stroke patients and hemorrhagic stroke. Mechanical phlebitis occurs in the adult and elderly age groups. The elderly age group experienced more phlebitis mechanics compared to adults.

This is in accordance with research conducted by (Abolfotouh, 2014) which states that there is no age difference in the incidence ofphlebitis. Patients aged 18-45 years and over 46 years are at risk of developing phlebitis. At the age of more than 45 years there is an increase in hypercoagulability which increases the occurrence of thrombus in the veins. Research conducted by Kawada (2018), states that age does not affect phlebitis.Phlebitis more common in hemorrhagic stroke patients who received nicardipine therapy with concentrations $>130 \mathrm{~g} / \mathrm{ml}$.

This could be due to the selection of the insertion site for peripheral infusion and infusion care. The insertion area in this study cannot be used as a research variable because there is no documentation of the infusion insertion area on the phlebitis surveillance sheet in medical records and SIMRS. Development of surveillance instruments phlebitis need to be equipped with a tick system insertion area and drawings for easy filling, evaluation and analysis.

In this study, there were differences in the sex factors of ischemic stroke and hemorrhagic stroke patients who experienced mechanical phlebitis. The incidence of mechanical phlebitis is more common in female stroke patients. Hemorrhagic stroke patients who are female have more mechanical phlebitis than male patients. The incidence of mechanical phlebitis in ischemic stroke is more experienced by male patients.

According to (Abadi, 2013) stated that gender did not affect phlebitis. The incidence of phlebitis is influenced by the insertion area, length of installation, and smoking habits. The insertion area in the dominant upper extremity is more at risk for phlebitis. The dominant extremity is used more for activities so that the cannula is easily shifted and irritates the endothelial wall of the vein. Installation time of 5-10 days is more at risk of experiencing phlebitis than installation of less than 5 days.

Gender characteristics affect the occurrence of phlebitis, this can be due to the length of infusion. The duration of infusion that is not well documented on the phlebitis surveillance form makes it difficult to analyze. It is necessary to develop a phlebitis surveillance form in the medical record file and SIMRS which is equipped with the length of installation and the reason for changing the infusion position so as to minimize bias and facilitate reporting of phlebitis events.
Table 1. Frequency distribution of respondent characteristics $(\mathrm{n}=92)$

\begin{tabular}{lcc}
\hline \multicolumn{1}{c}{ Variables } & Total & Percentage \\
\hline Type of Stroke & & \\
$\quad$ Ischemic & 56 & 60,9 \\
$\quad$ Hemorrhagic & 36 & 39,1 \\
Age & & \\
$\quad 36$ - 45 years & 4 & 4,3 \\
$\quad$ 46 - 55 years & 21 & 22,8 \\
56 - 65 year & 29 & 31,5 \\
$\quad>66$ years & 38 & 41,3 \\
Gender & & \\
$\quad$ Male & 38 & 41,3 \\
$\quad$ Female & 54 & 58,7 \\
History of Hypertension & & \\
$\quad$ Yes & 54 & 58,7 \\
$\quad$ No & 38 & 41,3 \\
History of Diabetes & & \\
Mellitus & & \\
$\quad$ Yes & 9 & 9,8 \\
$\quad$ No & 83 & 90,2 \\
Pneumonia & & \\
$\quad$ Yes & 4 & 4,3 \\
$\quad$ No & 88 & 95,6 \\
History of Stroke & & \\
$\quad$ Yes & 24 & 26,1 \\
$\quad$ No & 68 & 73,9 \\
\hline
\end{tabular}

Table 2. The results of the bivariate analysis of differences characteristics of ischemic and hemorrhagic stroke patients with mechanical phlebitis ( $\mathrm{n}=92$ )

\begin{tabular}{|c|c|c|c|c|c|}
\hline \multirow{3}{*}{ Variables } & \multicolumn{4}{|c|}{ Tipe Stroke } & \multirow{3}{*}{$\mathrm{p}$-value } \\
\hline & \multicolumn{2}{|c|}{$\begin{array}{l}\text { Ischemic } \\
\text { Stroke }\end{array}$} & \multicolumn{2}{|c|}{$\begin{array}{l}\text { Hemorrhagic } \\
\text { Stroke }\end{array}$} & \\
\hline & $\mathrm{n}$ & $\%$ & $\mathrm{n}$ & $\%$ & \\
\hline \multicolumn{6}{|l|}{ Age } \\
\hline $26-45$ & 2 & 50 & 2 & 50 & 0,511 \\
\hline$>46$ & 54 & 61,4 & 34 & 38,6 & \\
\hline \multicolumn{6}{|l|}{ Gender } \\
\hline Male & 29 & 76,3 & 9 & 23,7 & 0,020 \\
\hline Female & 27 & 50 & 27 & 50 & \\
\hline \multicolumn{6}{|l|}{$\begin{array}{l}\text { History of } \\
\text { Hypertension }\end{array}$} \\
\hline Yes & 28 & 51,9 & 26 & 48,1 & 0,058 \\
\hline No & 28 & 73,7 & 10 & 26,3 & \\
\hline \multicolumn{6}{|l|}{$\begin{array}{l}\text { History of } \\
\text { Diabetes }\end{array}$} \\
\hline \multicolumn{6}{|l|}{ Mellitus } \\
\hline Yes & 6 & 66,7 & 3 & 33,3 & 0,503 \\
\hline No & 50 & 60,2 & 33 & 39,8 & \\
\hline \multicolumn{6}{|l|}{ Pneumonia } \\
\hline Yes & 1 & 25 & 3 & 75 & 0,164 \\
\hline No & 55 & 62,5 & 33 & 37,5 & \\
\hline \multicolumn{6}{|l|}{$\begin{array}{l}\text { History of } \\
\text { Stroke }\end{array}$} \\
\hline Yes & 18 & 75 & 6 & 25 & 0,160 \\
\hline No & 38 & 55,9 & 30 & 36 & \\
\hline
\end{tabular}

There was no difference in the history of hypertension in ischemic stroke and hemorrhagic stroke patients who had mechanical phlebitis. Mechanical phlebitis occurs in patients who have a history of hypertension or not. The incidence of mechanical phlebitis is more common in stroke patients who have a history of hypertension. Hemorrhagic stroke patients who have a history of hypertension have more mechanical phlebitis than 
those who do not have a history of hypertension. The incidence of mechanical phlebitis in ischemic stroke patients who have a history of hypertension has the same proportion as patients without a history of hypertension.

The results of this study are in accordance with research conducted by (Salma, 2019) which states that phlebitis is more common in patients with hypertension compared to normotensive. Vascular endothelium in patients with hypertension may be damaged thereby increasing the incidence of phlebitis. In hypertension, there is vascular damage to the blood vessels which causes structural and functional changes in the blood which increase the risk of phlebitis because the vein wall is more easily traumatized at the time of insertion.

This could be due to the condition of the vein and pushing the cannula too hard and fast at the time of insertion. In the surveillance of phlebitis at SIMRS, there is no documentation of the condition of the veins and the number of times they have been inserted for infusion. The technique of insertion and stabilization of the infusion carried out by clinical nurse 1 with a short working period can increase the risk of mechanical phlebitis. It is necessary to develop phlebitis surveillance that is equipped with venous conditions and complicating factors for insertion. Workshop on infusion installation and stabilization techniques can improve the knowledge and skills of nurses to prevent and minimize the occurrence of mechanical phlebitis.

History of diabetes mellitus in ischemic stroke and hemorrhagic stroke patients who have mechanical phlebitis. The incidence of mechanical phlebitis was not influenced by a history of diabetes mellitus. Mechanical phlebitis is more common in patients who do not have a history of diabetes mellitus

The results of this study are in line with research conducted by Arias-Fernández, 2017 which states that phlebitis is more common in patients who do not have a history of diabetes mellitus. Phlebitis was more common in patients who underwent infusion with a cannula size larger than the diameter of the peripheral veins. Cannula larger than the diameter of the vein can traumatize the venous endothelium and cause phlebitis.

The characteristic history of immunosuppression did not affect mechanical phlebitis. This can occur due to the cannula material used at the time of peripheral infusion. Cannula made of vialon have a lower risk of mechanical phlebitis than cannula made of Teflon. In this study, the cannula material could not be used as a variable because the phlebitis surveillance sheet did not contain any components of the cannula material, so it was not possible to analyze the differences in the incidence of phlebitis based on the cannula material. It is necessary to develop a phlebitis surveillance sheet containing peripheral infusion cannula material.

The results of this study showed that there was no difference in the factors associated with infectious disease in ischemic stroke and hemorrhagic stroke patients who had mechanical phlebitis. Pneumonia was not associated with the incidence of mechanical phlebitis.

Mechanical phlebitis was more common in stroke patients who did not have pneumonia.

This is contrary to research conducted by (Salma, 2019) which states that phlebitis is more common in patients who have co-infectious diseases. Patients with infection receive intravenous antibiotic therapy. Injection of antibiotics via a port cannula can cause irritation of the venous endothelium which increases the risk of phlebitis. According to (Lv, 2020) in patients with infectious diseases the spread of microorganisms comes from septic emboli to the intravenous cannula, thereby increasing the risk of phlebitis. Patients who received antibiotics by infusion had a lower risk of developing phlebitis than patients who did not receive antibiotic therapy.

The characteristics of co-infectious diseases did not differ between ischemic stroke and hemorrhagic stroke patients who had mechanical phlebitis. This could be due to the patient's poor nutritional status. Patients with a body mass index that is less than normal have thin and fragile veins and a decreased immune system, which puts them at risk for mechanical phlebitis. Poor documentation of weight and height causes not being able to know body mass index. Compliance with documentation of weight and height when conducting nursing assessments is needed to determine the nutritional status of patients and nursing interventions. Completeness of data is required on the status of the patient's medical record which contains weight, height and BMI accompanied by supervision of compliance with nursing care documentation from assessment to nursing evaluation.

\section{CONCLUSION}

The results of this study indicate that there are differences in gender characteristics between ischemic stroke and hemorrhagic stroke patients who experience mechanical phlebitis. Characteristics of age, history of circulatory disorders, immunosuppression, co-infection, and stroke did not make any difference between ischemic stroke and hemorrhagic on the incidence of mechanical phlebitis. It is hoped that there will be development of phlebitis surveillance documents that are equipped with items of insertion area, cannula material, reasons for infusion release, venous condition, length of installation, frequency of insertion, observation of each shift accompanied by controlling documentation of phlebitis surveillance and nursing care, supervision of compliance with infusion bundle installation and care.

\section{REFERENCE}

Abadi, P., 2013. Investigating role of mechanical and chemical factors in the creation of peripheral vein in flammarion in hospitalization patients in 
hospital in Zahedan, Iran. Life Science Journal, 10(SUPPL.1), pp. 379-383

Abolfotouh, M. A., 2014. Prospective study of incidence and predictors of peripheral intravenous catheter-induced complications. Therapeutics and Clinical Risk Management, pp. 993-1001.

Arias-Fernández, L., 2017. Incidence and risk factors of phlebitis associated to peripheral intravenous catheters. Enfermería Clínica (English Edition), 27(2), pp. 79-86.

Blanco-Mavillard, I., 2019. Incidence of peripheral intravenous catheter failure among inpatients: Variability between microbiological data and clinical signs and symptoms. Antimicrobial Resistance and Infection Control, pp. 1-11.

Gorski, L. A., 2016. Infusion Therapy Standards of Practice. Journal of Infusion Nursing, pp. S95- S98.

Kawada, K., 2018. Reduction of Nicardipine-Related Phlebitis in Patients with Acute Stroke by Diluting Its Concentration. Journal of Stroke and Cerebrovascular Diseases, 27(7), pp. 1783-1788

Lv, L., 2020. The incidence and risk of infusion phlebitis with peripheral intravenous catheters: A meta-analysis. Journal of Vascular Access, pp. 342349

Maier, D., 2019. To Replace or Not to Replace? Replacing Short Peripheral Catheters Based on Clinical Indication. Journal of Infusion Nursing, 42(3), pp. 143-148

Phillips, L. D. \& Gorski, L. A., 2014. Manual of I.V Therapeutics Evidence-Based Practice for Infusion Therapy 6th Edition. Philadelphia: F.A.Davis Company.
Salma, U., 2019. Frequency of peripheral intravenous catheter related phlebitis and related risk factors: A prospective study. Journal of Medicine (Bangladesh), 20(1), pp. 29-33.

Simin, D., 2019. Incidence, severity and risk factors of peripheral intravenous cannula-induced complications: An observational prospective study. Journal of Clinical Nursing, pp. 1585-1599.

Siregar, A. W., 2019. Effectiveness of ADULT Bundle to Prevention of Phlebitis. Journal of Nursing and Health Science, pp. 76-80.

Torres-Aguila, N. P., 2019. Clinical variables and genetic risk factors associated with the acute outcome of ischemic stroke: A systematic review. Journal of Stroke, pp. 276-289

Unahalekhaka, A., 2016. Epidemiology of HealthcareAssociated Infections, international federation of infection control, 3rd edition. In: Basic Concepts of Infection Control. UK: International Federation Of Infection Control, pp. 1-9.

Ying, C. X., 2020. Perceptions of risk factors for phlebitis among Malaysian nurses. British Journal of Nursing, 29(2), pp. S18-S23. 